\title{
On the nature of the unidentified X-ray $/ \gamma$-ray sources IGR J18027-1455 and IGR J21247+5058
}

\author{
J.A. Combi (jcombi@ujaen.es) \\ Universidad de Jaén \\ Instituto Argentino de Radioastronomía (IAR) \\ M. Ribó \\ Service d'Astrophysique, CEA Saclay \\ I.F. Mirabel \\ Service d'Astrophysique, CEA Saclay \\ Instituto de Astronomía y Física del Espacio (IAFE)
}

\begin{abstract}
We present a multiwavelength study of the environment of the unidentified X-ray/ $\gamma$-ray sources IGR J18027-1455 and IGR J21247+5058, recently discovered by the IBIS/ISGRI instrument, onboard the INTEGRAL satellite. The main properties of the sources found inside their position error circles, give us clues about the nature of these high-energy sources.
\end{abstract}

Keywords: X-ray:galaxies, radio continuum:galaxies, infrared:galaxies

\section{Introduction}

Since it began to operate in November 2003 until the time of writing (about 18 months), the IBIS/ISGRI instrument onboard the INTEGRAL satellite (Winkler et al. 2003) has discovered up to 40 unidentified X-ray/ $\gamma$-ray sources in the energy range from 20 to $100 \mathrm{keV}$ (a regularly updated list is kept in http://isdc. unige.ch/ rodrigue/html/ /igrsources.html). These sources can be high or low mass X-ray binaries (not detected previously due to the absence of hard X-ray surveys), isolated pulsars, clusters of galaxies or AGNs, among other objects. New observations along the electromagnetic spectrum are necessary to discern their nature.

In this work, we present a multiwavelength study of the fields containing the unidentified INTEGRAL sources IGR J18027-1455 and IGR J21247+5058 (Walter et al. 2004), as earlier done in Combi et al. (2004) and in Ribó et al. (2004) but using here the new positions and information reported in Bird et al. (2004). Based on the properties of the sources found at other frequencies inside the error circles in position of the IGR sources, we suggest possible origins for both high-energy sources.

(c) 2018 Kluwer Academic Publishers. Printed in the Netherlands. 


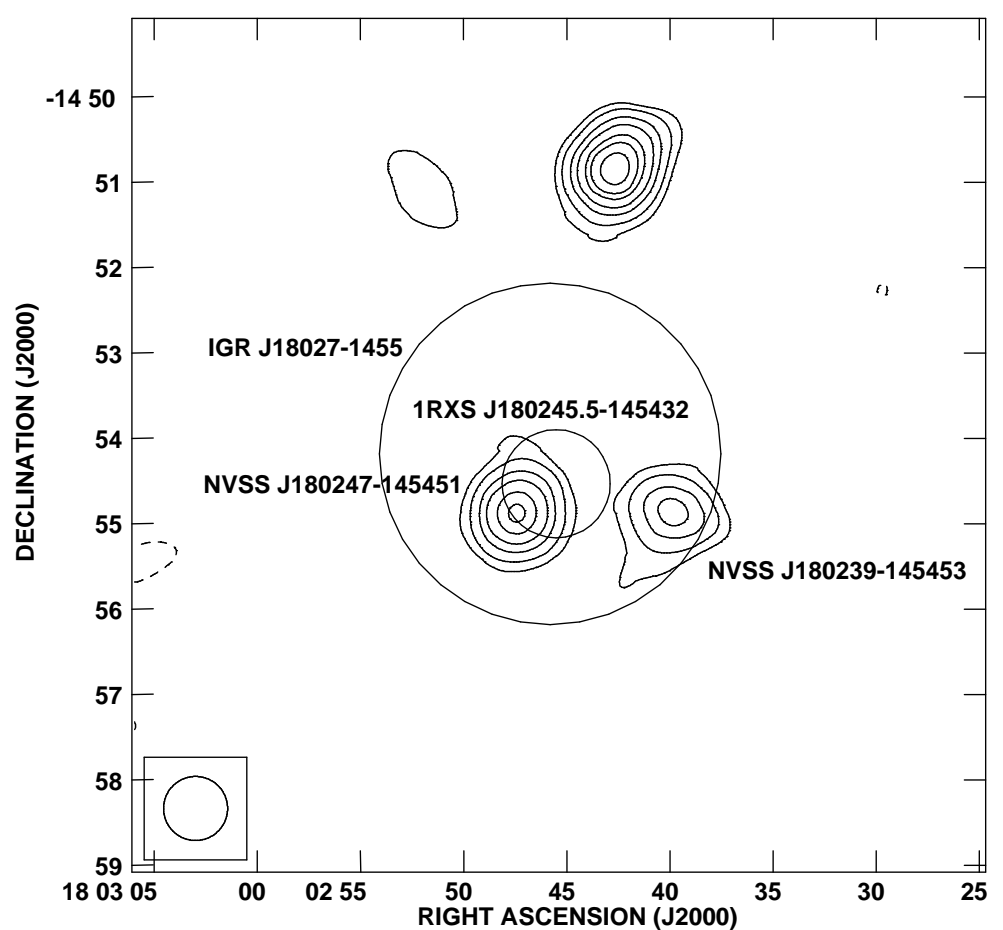

Figure 1. Image of the NVSS data obtained with the VLA at $1.4 \mathrm{GHz}$ on 1997 October 13 around IGR J18027-1455. The image size is $10^{\prime} \times 10^{\prime}$. Contours represent $-3,3,5,8,11,15,18$, and 22 times the rms noise level of $0.5 \mathrm{mJy}^{\text {beam }}{ }^{-1}$. The circle in the bottom left corner represents the 45 arcsec of Full Width at Half Maximum (FWHM) of the convolving beam. Two NVSS sources fall inside the $90 \%$ error circle in position of IGR J18027-1455, and one of them is within the $2 \sigma$ uncertainty error circle of a ROSAT source.

\section{The IGR J18027-1455 field}

The source IGR J18027-1455 was detected with a significance of $8.9 \sigma$ in the energy range from 20 to $100 \mathrm{keV}$ during $769 \mathrm{ksec}$ of observations conducted within the INTEGRAL/IBIS survey of the Galactic plane (Bird et al. 2004). The obtained fluxes compared to the Crab are $F_{(20-40 \mathrm{keV})}=2.6 \pm 0.2 \mathrm{mCrab}$ and $F_{(40-100 \mathrm{keV})}=3.0 \pm 0.4 \mathrm{mCrab}$. Within its 2 arcmin-radius position error circle (at $90 \%$ confidence or $1.6 \sigma$ uncertainty $)$, centered at $(\alpha, \delta)_{\mathrm{J} 2000.0}=\left(18^{\mathrm{h}} 02^{\mathrm{m}} 45^{\mathrm{s}} 8,-14^{\circ} 54^{\prime} 11^{\prime \prime}\right)$, we have found two weak point-like radio sources in the $1.4 \mathrm{GHz}$ NRAO VLA Sky Survey (NVSS, Condon et al. 1998) maps (see Fig. 1). The first one, the source NVSS J180239-145453, has an estimated position of $(\alpha, \delta)_{\mathrm{J} 2000.0}=\left(18^{\mathrm{h}} 02^{\mathrm{m}} 39^{\mathrm{s}} .94 \pm 0.25,-14^{\circ} 54^{\prime} 53^{\prime \prime} \cdot 6 \pm 3^{\prime \prime} \cdot 3\right)$ ( $1 \sigma$ uncertainties), has a flux density of $6.9 \pm 0.6 \mathrm{mJy}$ and has no near infrared (NIR) counterpart in the 2MASS catalog (Cutri et al. 2003). 


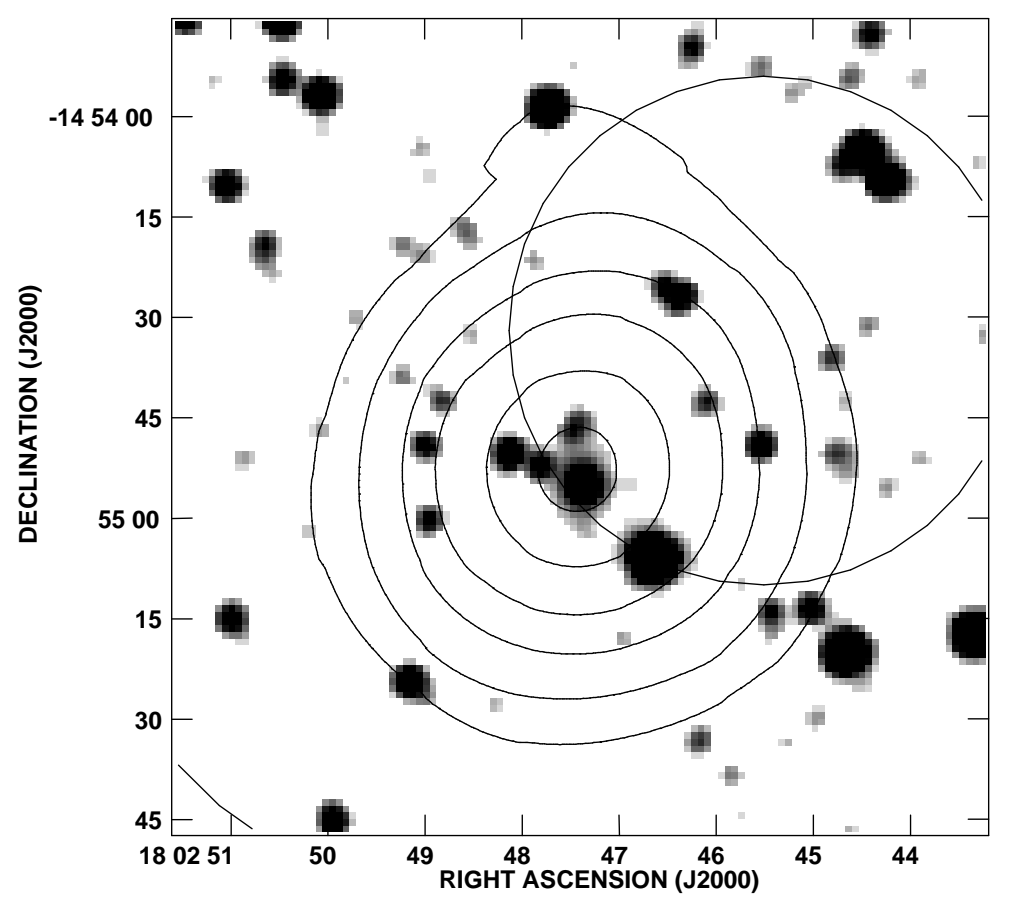

Figure 2. Enlargement of a $2^{\prime} \times 2^{\prime}$ region of Fig. 1 around NVSS J180247-145451, where we plot in greyscale the $2 \mathrm{MASS} K_{\mathrm{s}}$-band image. The extended NIR source 2MASXi J1802473-145454 is clearly visible in a position compatible with the peak of the radio source.

The second one, NVSS J180247-145451, has an estimated position of $(\alpha, \delta)_{\mathrm{J} 2000.0}=\left(18^{\mathrm{h}} 02^{\mathrm{m}} 47^{\mathrm{s}} .37 \pm 0.13,-14^{\circ} 54^{\prime} 51^{\mathrm{s}} .6 \pm 22^{\prime \prime} 2\right)$, a flux density of $10.5 \pm 0.6 \mathrm{mJy}$, and lies inside and near the edge of the $2 \sigma$ position error circle of the faint ROSAT X-ray source 1RXS J180245.5-145432 (Voges et al. 2000), which is well within the IBIS/ISGRI error circle. Inside the $2 \sigma$ position error ellipse of this radio source, it is located an extended NIR source, 2MASXi J1802473-145454 (see Fig. 2), which has coordinates $(\alpha, \delta)_{\mathrm{J} 2000.0}=\left(18^{\mathrm{h}} 02^{\mathrm{m}} 47^{\mathrm{s}} .370 \pm 0.002,-14^{\circ} 54^{\prime} 54^{\prime \prime} \cdot 76 \pm\right.$ $\left.00^{\prime \prime} 03\right)$ and magnitudes $J=13.18 \pm 0.07, H=12.02 \pm 0.09, K_{\mathrm{s}}=10.94 \pm 0.04$. The optical counterpart of this NIR source has average magnitudes $B=19.3 \pm 1.0, R=14.9 \pm 0.8$ and $I=13.8 \pm 0.5$ in the USNO-B1.0 catalog (Monet et al. 2003). The photometry of the NIR/optical counterpart is not consistent with a stellar spectrum. Optical and X-ray observations are planned to confirm the extragalactic nature of the source and to study its spectrum at softer X-rays. 


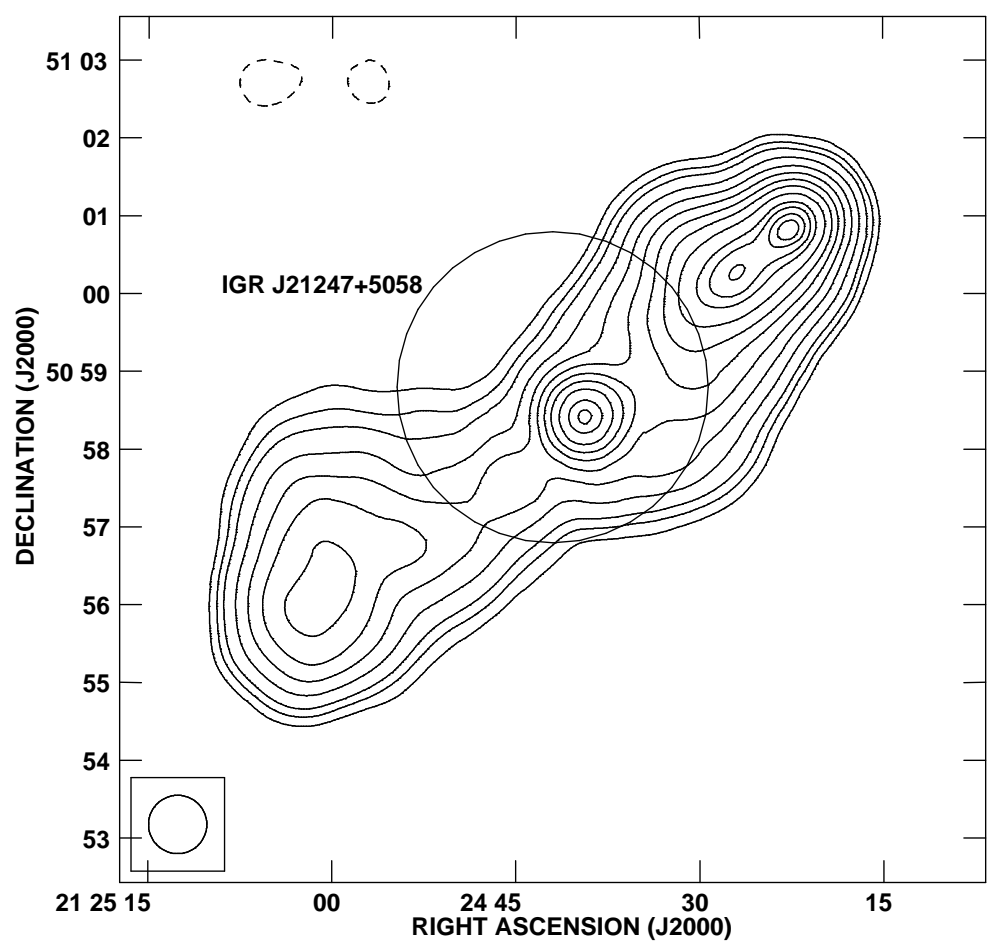

Figure 3. Image of the NVSS data obtained with the VLA at $1.4 \mathrm{GHz}$ on 1995 April 3 around IGR J21247+5058. The image size is $10^{\prime} \times 10^{\prime}$. The core of the radio source 4C 50.55 is well within the $90 \%$ uncertainty error circle of the IGR source. Contours are $-3,3,6,10,20,35,55,80,110,150,200,230,260$ and 280 times the rms noise level of $1 \mathrm{mJy}_{\text {beam }}{ }^{-1}$. The circle in the bottom left corner represents the $45^{\prime \prime}$ FWHM of the convolving beam.

\section{The IGR J21247+5058 field}

This source was detected with a significance of $6.5 \sigma$ in the energy range from 20 to $100 \mathrm{keV}$ during only $70 \mathrm{ksec}$ of observations conducted within the same INTEGRAL/IBIS survey of the Galactic plane (Bird et al. 2004). The obtained fluxes compared to the Crab are $F_{(20-40 \mathrm{keV})}=5.4 \pm 0.7 \mathrm{mCrab}$ and $F_{(40-100 \mathrm{keV})}=9.3 \pm 1.4 \mathrm{mCrab}$. Inside its 2 arcmin-radius position error circle (at $90 \%$ confidence or $1.6 \sigma$ uncertainty), centered at $(\alpha, \delta)_{\mathrm{J} 2000.0}=\left(21^{\mathrm{h}} 24^{\mathrm{m}} 42^{\mathrm{s}} .0,+50^{\circ} 58^{\prime} 48^{\prime \prime}\right)$ is located the core of the bright radio source 4C 50.55 (see Fig. 3), also known as GPSR 93.3194+0.394, KR2, NRAO 659 or BG 2122+50, among other names.

The core has a flat radio spectrum with a peak flux density of $237 \mathrm{mJy}$ beam $^{-1}$ at $1.4 \mathrm{GHz}$. It is at the center of an elongated structure of $10 \times 3$ arcmin (see Fig. 3) that ends in two large radio lobes having 


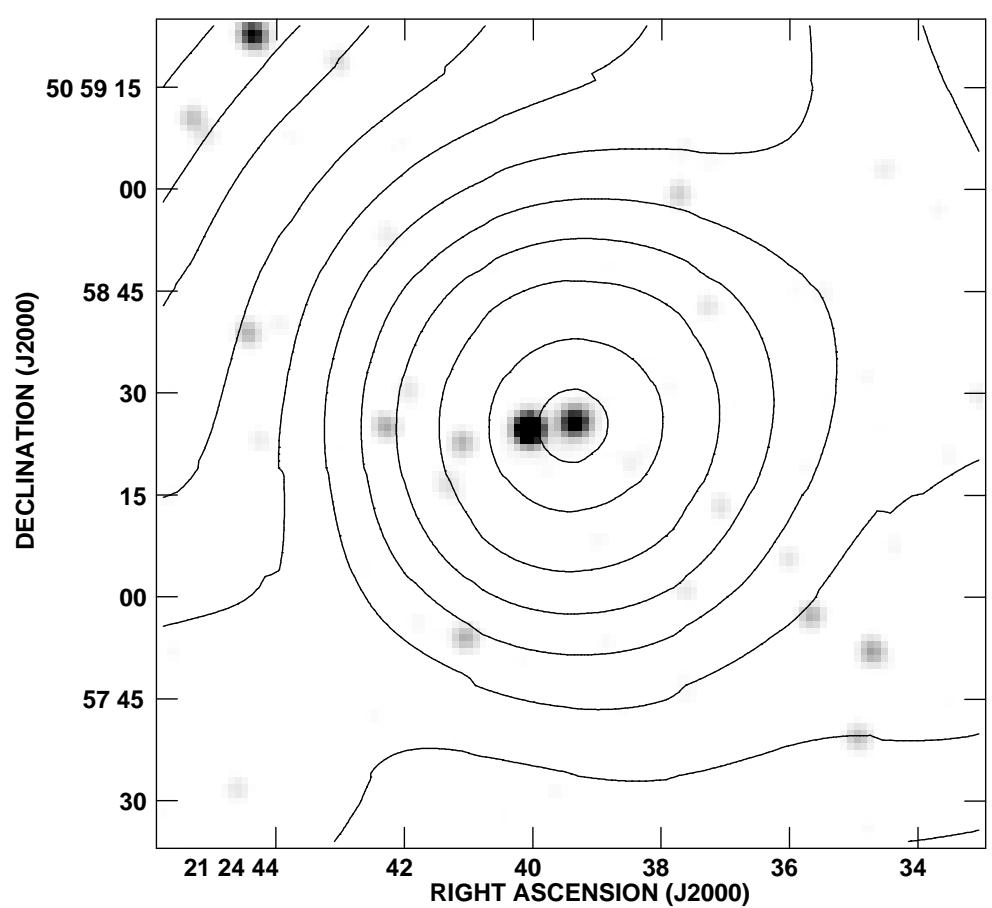

Figure 4. Enlargement of a $2^{\prime} \times 2^{\prime}$ region of Fig. 3 around the core of $4 \mathrm{C} 50.55$, where we plot in greyscale the $2 \mathrm{MASS} K_{\mathrm{s}}$-band image showing the proposed NIR counterpart, coincident with the radio peak.

peak flux densities of 288 mJy beam ${ }^{-1}$ (NW) and 92 mJy beam ${ }^{-1}$ (SE), and spectral indices $\alpha \sim-0.6$ (where $S_{\nu} \propto \nu^{+\alpha}$ ), compatible with optically thin synchrotron radiation (Mantovani et al. 1982). The morphology of $4 \mathrm{C} 50.55$ is typical of a radio galaxy, and the comparison among published radio data shows no significant changes in flux and morphology during the last 30 years (see, e.g. Fanti et al. 1981). These properties are consistent with an extragalactic source, although, to our knowledge, no conclusive optical counterpart of a galaxy host has ever been reported, probably due to its location close to the Galactic plane $\left(l=+93.32^{\circ}, b=+0.39^{\circ}\right)$.

The estimated position of the radio core from the NVSS map and data in the literature is $(\alpha, \delta)_{\mathrm{J} 2000.0}=\left(21^{\mathrm{h}} 24^{\mathrm{m}} 39.35 \pm 0.03,+50^{\circ} 58^{\prime} 25^{\prime \prime} .8\right.$ \pm 0 "' 2 ) ( $68 \%$ or $1 \sigma$ uncertainty). Coincident with this position there is the NIR source 2MASS J21243932+5058259 (see Fig. 4), with coordinates $(\alpha, \delta)_{\mathrm{J} 2000.0}=\left(21^{\mathrm{h}} 24^{\mathrm{m}} 39.328 \pm 0.003,+50^{\circ} 58^{\prime} 25^{\prime \prime} \cdot 93 \pm 00^{\prime \prime} 03\right)(68 \%$ or $1 \sigma)$, and magnitudes $J=13.27 \pm 0.04, H=12.38 \pm 0.06, K_{\mathrm{s}}=11.37 \pm 0.04$. The optical counterpart of this NIR source has average magnitudes $B=16.9 \pm 0.2$ and $R=15.1 \pm 0.2$ in the USNO-B1.0 catalog (Monet et al. 2003). The photometry of the NIR/optical counterpart is not consistent 
with a stellar spectrum. There is no catalogued ROSAT or EGRET source coincident with IGR J21247+5058. Optical and X-ray observations are planned to confirm the extragalactic nature of the source and to study its spectrum at softer X-rays.

\section{Further Comments}

We have presented a multiwavelength study of the emission towards the direction of two recently discovered INTEGRAL sources, namely IGR J18027-1455 and IGR J21247+5058. Inside the position error box of the first one, we have found the radio source NVSS J180247-145451, compatible with the soft X-ray source 1RXS J180245.5-145432, and having the extended source 2MASXi J1802473-145454 as NIR counterpart. We suggest that all these sources are likely associated with the counterpart of the hard X-ray source. The photometry of the NIR/optical source does not agree with a stellar spectrum, hence this object seems to be of extragalactic nature. This is supported by the extended appearance of the NIR counterpart. In the second case, we have found that the core of an extended radio source, which ends in two large radio lobes, lies well within the position error circle of the INTEGRAL source. The morphology of this extended source is typical of a radio galaxy. Coincident with the position of the radio core is a 2MASS source, with an optical counterpart not compatible with a stellar spectrum.

If the X-ray emission of IGR J21247+5058 comes indeed from a radio galaxy, then it should originate in the core and/or in the first secction of the jet, where synchrotron or inverse Compton emission processes dominate (Harris \& Krawczynski 2002). This source could be a similar case to 3C 273 (Marshall et al. 2001), where the detected X-rays fade along the jet. Evidence supporting this possibility was also presented by Harris et al. (2002) for the radio galaxy M84. In this object, these authors found an excess of X-ray emission, or knots, aligned with one of the radio jets. These knots lie at $\sim 300 \mathrm{pc}$ from the core and they are coincident with enhanced radio emission (see their Fig. 1), suggesting that the X-rays are produced by a synchrotron mechanism.

Recently, Bassani et al. (2004), have presented a study of extragalactic sources detected by INTEGRAL during its first year of operation.

Among them, 10 are active galaxies and one is a cluster of galaxies. If the extragalactic nature of the sources studied here is confirmed, then many of the unidentified INTEGRAL sources detected till now could increase considerably the number of extragalactic objects shining in the hard X-ray domain. 
Finally, it is interesting to note that there is no catalogued EGRET source coincident with these sources. Multiwavelength observations are currently in progress to unveil the nature of these high-energy sources.

\section{Acknowledgements}

We acknowledge Josep Martí for useful discussions. J.A.C. is a researcher of the programme Ramón y Cajal funded by the Spanish Ministery of Science and Technology and the University of Jaén, and was also supported by CONICET (under grant PEI 6384/03). M.R. acknowledges support by a Marie Curie Fellowship of the European Community programme Improving Human Potential under contract number HPMF-CT-2002-02053, and partial support by DGI of the Ministerio de Ciencia y Tecnología (Spain) under grant AYA2001-3092, as well as partial support by the European Regional Development Fund (ERDF/FEDER). This research has made use of the NASA's Astrophysics Data System Abstract Service, of the SIMBAD database, operated at CDS, Strasbourg, France, and of the NASA/IPAC Extragalactic Database (NED) which is operated by the Jet Propulsion Laboratory, California Institute of Technology, under contract with the National Aeronautics and Space Administration. The Digitized Sky Survey was produced at the Space Telescope Science Institute under U.S. Government grant NAG W-2166. This publication makes use of data products from the Two Micron All Sky Survey, which is a joint project of the University of Massachusetts and the Infrared Processing and Analysis Center/California Institute of Technology, funded by the National Aeronautics and Space Administration and the National Science Foundation.

\section{References}

Bassani, L., Malizia, A., Stephen, J. B., et al. 2004, in Proceedings of The 5th INTEGRAL Workshop: The Integral Universe, ESA SP-552, in press [arXiv:astro-ph/0404442]

Bird, A. J., Barlow, E. J., Bassani, L., et al. 2004, ApJ, 607, L33

Combi, J. A., Ribó, M., \& Mirabel, I. F. 2004, ATel, 246

Condon, J. J., Cotton, W. D., Greisen, E. W., et al. 1998, AJ, 115, 1693

Cutri, R. M., Skrutskie, M. F., van Dyk, S., et al. 2003, VizieR Online Data Catalog, II/246 (http://cdsweb.u-strasbg.fr/viz-bin/Cat?II/246)

Fanti, C., Mantovani, F., \& Tomasi, P. 1981, A\&AS, 43, 1

Harris, D. E., \& Krawczynski, H. 2002, ApJ, 565, 244

Harris, D. E., Finoguenov, A., Bridle, A. H., Hardcastle, M. J., \& Laing, R. 2002, ApJ, 580, 110 
Mantovani, F., Nanni, M., Salter, C. J., \& Tomasi, P. 1982, A\&A, 105, 176

Marshall, H. L., Harris, D. E., Grimes, J. P., et al. 2001, ApJ, 549, L167

Monet, D. G., Levine, S. E., Canzian, B., et al. 2003, AJ, 125, 984 (http://cdsweb.u-strasbg.fr/viz-bin/Cat?I/284)

Ribó, M., Combi, J. A., \& Mirabel, I. F. 2004, ATel, 235

Voges, W., Aschenbach, B., Boller, Th., et al. 2000, VizieR Online Data Catalog, IX/29 (http://cdsweb.u-strasbg.fr/viz-bin/Cat?IX/29)

Walter, R., Bodaghee, A., Barlow, E., et al. 2004, ATel, 229

Winkler, C., Courvoisier, T. J.-L., Di Cocco, G., et al. 2003, A\&A, 411, L1

Address for Offprints: Jorge A. Combi

Departamento de Física

Escuela Politécnica Superior

Universidad de Jaén

Virgen de la Cabeza 2

E-23071 Jaén

Spain 